\title{
Is There a Role for Platelet-Rich Plasma Injection in Pediatrics? a Narrative Review
}

\author{
Jorge Benito ${ }^{1}$, Phong Truong ${ }^{1}$ Eric Bradley ${ }^{1}$, Cindy Ho $^{1}$ and Cameron McLaury ${ }^{2}$ \\ ${ }^{1}$ Department of Orthopedic Surgery, Larkin Community Hospital, USA \\ ${ }^{2}$ College of Medicine, Oklahoma University Health Science Center, USA
}

*Corresponding author: Jorge Benito, Department of Orthopedic Surgery, Larkin Community Hospital, 7000 SW 62nd Ave, Suite 401, South Miami, FL 33143, USA

\begin{abstract}
This review identifies some of the relevant literature regarding the application of platelet-rich plasma (PRP) and the corresponding evidence of its use in the pediatric population. Because of its potential in various regenerative processes, there is a building interest in its utilization. However, very few high-quality studies investigating the therapeutic use of PRP exist for many reasons. The concentration of plasma constituents, response to PRP in different tissues, and its preparation are some of the issues that have been overlooked or not standardized when investigating its efficacy. With this lack of evidence, PRP's utilization in the general population, and consequently in pediatrics, has been under-investigated and relegated mostly to animal models. We postulate that PRP may have some of its best utility in the young athlete with musculoskeletal derangements. In addition, further investigations need to be conducted in pediatric patients to determine whether PRP will be beneficial.
\end{abstract}

Keywords: Orthobiologics; Platelet rich plasma (PRP); Pediatrics, Sports injuries; Sports medicine; Orthopedic surgery

Abbreviations: (PRP): Platelet rich plasma; (ACL): Anterior cruciate ligament; (UCL): Ulnar collateral ligament; (MCL): Medial collateral ligament; (MPFL): Medial patellofemoral ligament; (RICE): Rest ice compression and elevation; (DASH): Disabilities of the Arm Shoulder and Hand; (KJOC): Kerlan-Jobe Orthopaedic Clinic Shoulder and Elbow

\section{Introduction}

Over the last three decades, there has been increasing interest in biological treatments for musculoskeletal pathology. Advances in plasma and stem cell research have led to the study and utilization of our own biologic machinery's regenerative or reparative potential. Uses of these therapies have their roots in plastic surgery starting in the 1990s, and since that time, there has been an increased focus on their use in sports medicine [1] The theory behind platelet-rich plasma (PRP) is the sequestration of blood plasma, which contains many of the healing factors responsible for tissue repair, to the area in question. This article aims to review PRP as a treatment modality and identify the most common uses of PRP in the pediatric population. We will also bring attention to its current limitations and future potential. There is a wealth of studies on its use in several sports-related pathologies, but studies on pediatric use and indications are still lacking [2]

\section{What is PRP}

Traditionally, PRP has been defined depending on its most abundant constituent, and fibrin density once activated.3 The four major types are Pure Platelet-Rich Plasma (P-PRP) (or LeukocytePoor Platelet-Rich Plasma (LP-PRP)), Leukocyte-and Platelet-Rich Plasma (LR-PRP), Pure Platelet-Rich Fibrin (P-PRF) (or LeukocytePoor Platelet-Rich Fibrin, and Leukocyte-and Platelet-Rich Fibrin (L-PRF)] [3] Each of these preparations has its specific composition, which has indications depending on the pathology in question. Each formulation induces specific effects on cartilage depending on the concentration of different factors. However, one of the significant difficulties with the administration of PRP products is utilizing the correct concentration or ratio of plasma factors that will induce the appropriate response [4]. For instance, in a 2015 study by Zhou, both L-PRP and P-PRP demonstrated different responses to 
the differentiation of trophoblast stem cells to tenocytes. L-PRP was detrimental to these tenocytes by activating catabolic effects on tendons. On the other hand, P-PRP was anabolic to tenocytes, leading to healing via scar tissue formation [5].

Current literature has recognized some evidence for the use of PRP depending on the pathology in question. In a review by Le, there is support for the use of LR-PRP for lateral epicondylitis, osteoarthritis in the knee, patella tendonitis, plantar fasciitis, and as an adjunct for pain following bone-patellar tendon-bone ACL reconstruction. Alternatively, PRP is also shown to be ineffective in Achilles tendinitis, injured muscle, fracture non-unions, and ACL or Achilles tendon repair. Furthermore, there is incomplete evidence for PRP in rotator cuff tears, osteoarthritis of the hip, and ankle sprains [6]. Some of the criticism from lack of evidence or utility for PRP is due to the varying methodology of PRP preparation and formulation across studies. There is little to no clear literature on the active components responsible for the bulk of the biologic response that PRP theoretically induces. We are aware of these molecules and the responses they impart. However, the concentration and heterogeneity between samples obtained from different patients vary. This diversity between formulations of PRP can potentially produce varying results from case to case [7].

\section{Uses for PRP}

Regardless of the heterogeneity with PRP's formulations, it can be appreciated that cytokines and PRP factors may be responsible for its regenerative properties. There is evidence that these factors may very well expedite healing, especially in chronic tendinopathies, where the tendon in question lacks the nutrient environment to repair itself [6] A systematic review and metaanalysis by Miller concluded that PRP was effective in treating and expediting the healing in these aforementioned chronic conditions [8] Other indications for PRP's use in the literature review include but are not limited to rotator cuff repair, Achilles tendon repair, and ACL surgery. Sprains and muscle injuries have also been a subject of interest. Moreover, PRP has been studied for use in other non-soft tissue-related pathologies, including osteoarthritis of hip and knee and fracture management and nonunions. [6] It must be mentioned that the majority of randomized controlled trials testing the efficacy of PRP are not high quality. Furthermore, to achieve better studies and obtain reproducible results, Chahla, et al. published the importance of standardizing PRP harvesting preparations and PRP compositions to make comparisons between studies.

Further emphasizing the issue of timing and formulation of a PRP injection, Laprade, et al. investigated whether or not the use of a single dose of varying concentrations of PRP accelerates ligament healing and improves the histological characteristics and biomechanical properties with a comparison to standard saline injections on the contralateral limb [9] The study was conducted by inducing a grade 3 MCL sprain on New Zealand White rabbits, and then an injection of either saline or varying concentrations of PRP was delivered immediately post-operatively. The study concluded that low dose concentrations of PRP injections into injured ligaments did not improve the healing of injured MCLs, and higher doses seemed to decrease the quality of the ligament healing. The study further emphasized that additional in vivo studies are needed to standardize the timing of injection and the concentration of PRP injection in the treatment of ligament injuries [9].

\section{Use in pediatrics}

With the advent of PRP use in adults, there is gaining interest in its use for pediatric sports injuries. The following points call attention for further investigation when considering PRP in the young athlete: increasingly promising data with more randomized controlled trials, a better understanding of the healing factors found in plasma, improved protocols for obtaining specific formulations, and reducing heterogeneity among said formulations. Furthermore, we should note the potentially increased efficacy among the younger population due to genetic factors, the negative immunogenicity with autologous PRP, and the non-invasive nature of these treatments. With the increasing number of sports injuries in the younger population, research in biological treatment is imperative. Schroeder et al. investigated overuse injuries from 2006 to 2012 and found these injuries occurred in 1.50 per 10,000 athletic exposures within the interest population. Females had a greater risk of overuse versus males, with the most significant risk being in track and field. $7.7 \%$ of all injuries in this population were overuse injuries. The most frequent site was in the lower leg. Injuries most frequently resulted in less than a week of time off, with $7.6 \%$ of the patients reporting time loss greater than three weeks [10].

Similarly, there is an increased amount of ligamentous injuries necessitating reconstruction in the young population. According to some studies, ACL injuries requiring reconstruction alone have increased 924\% from 1994 to 2006 [11] This study identified prolonged sports participation, increased amount of athletes participating, and a focus on a single sport as risk factors for the increased incidence. Other common sports injuries in the pediatric population include the medial patellofemoral ligament (MPFL) and the ulnar collateral ligament (UCL) [2] There are currently no studies examining PRP's use as an augment for MPFL injury or repair. Other indications presented by Bray, et al. that warrant further study include PRP for tendon injuries, cartilage defects, and fractures, although even less data is available for these situations. The majority of studies investigating PRP's potential in these instances continue to be limited in number, contradictory in results, and limited to adult subjects. 


\section{Potential Benefits of PRP in pediatrics}

With increasing sports participation and focus on specialization in a single sport with minimal rest in training, overuse injuries in adolescent athletes are increasing. These injuries vary in morbidity from requiring rest for a few days and subsequently missing a few games or practices to season-ending injuries. While rest, ice, compression, elevation (RICE therapy) with or without accompanying physical therapy are often chosen as treatment regimens, there is a lack of level I evidence to support their efficacy.12 While not performed in pediatric patients but rather patients $>/=$ 18 years of age with a median age of 21 years old, a randomized controlled trial by Hamid, et al. in 2014 investigated the effect of a single PRP injection and a standardized rehab protocol versus a rehabilitation program alone on grade 2 hamstring injuries. The primary outcome of return to play was found to be statistically significant ( $\mathrm{p}=.02$ ), and substantially sooner with the PRP injection with an average return to play of 26.7 days + /- 7.0 days versus 42.5 +/- 20.6 days in the control group [13]

A systematic review by Figueroa et al. compared ACL reconstruction with PRP as an adjunct versus ACL reconstruction alone. In total, eleven studies were reviewed. Six of those demonstrated statistical significance in regards to "graft maturation" with PRP. One study showed improved tunnel healing and another showed better clinical outcomes. Five studies showed no added benefit when PRP was added to ACL reconstruction [14] Podesta, et al. conducted a case series in 2013 on 34 athletes with partialthickness UCL tears as evidenced by clinical examination and confirmed on MRI, who failed at least two months of nonoperative care. The patients were treated with a single leukocyte rich PRP injection. The average age of these players was 18 years, with a range from 14 to 34 years. The study resulted in 30 out of 34 patients returning to the same level of play without issues at an average of 12 weeks post-treatment. The average Disabilities of the Arm, Shoulder and Hand (DASH) score improved from 21 to 1 , and the average Kerlan-Jobe Orthopaedic Clinic Shoulder and Elbow (KJOC) score improved from 46 to 93-both of which were statistically significant. The study concluded that PRP was effective in treating partial UCL tears of the elbow in young athletes [15]

On the contrary, Chauhan, et al. conducted a retrospective study on PRP injections in 133 MLB pitchers versus no PRP in 411 MLB pitchers in the nonoperative treatment of UCL injuries. The PRP group resulted in a significantly longer return to throwing and a significantly longer return to play. However, PRP's formulation was non-standardized, with some patients receiving leukocyte rich PRP and others receiving leukocyte poor injections. Additionally, the time from injury to injection was not standardized. Notably, the average age of the non-PRP group was 22.6 years old versus 24.3 years old for the PRP group. [16] However, as evidenced by
Podesta, et al. PRP's efficacy was seen in young athletes with PRP. While the two studies' results differ in their outcomes, both suggest younger patients can do well with the nonoperative case of UCL injuries. The two studies differed on major criteria such as time from injury to injection and type of PRP injections. Therefore, further investigation needs to be conducted on the type of injection and the time from injury to injection, both of which need to be standardized for further conclusions, which seems to be one of the underlying problems with PRP studies. Additionally, since younger patients have a greater healing capacity, an appropriately timed PRP injection of the correct formulation may further enhance their recovery and return to sport.

Tendinopathies are another indication for the potential benefit of PRP administration. Sanchez, et al. injected PRP during Achilles tendon repair in a case-control study from 2007. In the participants treated with PRP, return to base function was faster than in the non-PRP group [17] Kon, et al. found significant improvement in all functional scores used in their study when PRP was used to treat jumper's knee (patellar tendinitis) [18]. One of the most common injuries to young athletes is chondral damage. Chondral injury is another area of interest as it is a common injury with the potential for arthritis development. Many treatments have been developed to treat cartilage lesions, including microfracture, which is a similar concept to PRP. By surrounding the defective cartilage with a nutrient-rich environment, microfracture and PRP theoretically lead to chondral repair via similar mechanisms. Again, Bray et al. suggest future investigation into PRP as a therapeutic for these injuries; however, at this time, these injuries are mostly treated with microfracture, osteochondral autograft, osteochondral allografts, and autologous chondrocyte implantation. Good results have been documented in the pediatric population. One of the reasons why PRP use in this group is possibly incomplete is the more urgent nature of treating these injuries, which can accelerate towards arthritis.

Finally, fracture management is another area where PRP administration has piqued some interest. One study demonstrated reduced clinical and radiographic healing time of femoral neck fractures with PRP in addition to cannulated screw fixation compared to just cannulated screw fixation $[19,20]$ However, a subsequent paper demonstrated the opposite effect. Singh reports that although PRP may provide an artificial hematoma effect in the initial healing phase, it does not affect femoral shaft fracture healing when used with intramedullary nailing.

\section{Conclusion}

Young athletes often participate in year-round sports. These athletes are also focusing on single sports at a younger age, and therefore participation in multiple sports and appropriate crosstraining is often lacking. Without consistent rest periods or cross- 
training, an increasing number of athletes will continue to sustain overuse and sports-related injuries. Since younger patients have a greater healing capacity, adding PRP to the treatment regimen, in theory, may enhance their ability to return to their sport sooner. While PRP's potential applications in sports medicine are still being studied extensively, no trials are specifically studying pediatric patients. Since cytokines found in PRP are involved in the signaling pathways that occur during healing stages of inflammation, cellular proliferation, and subsequent tissue remodeling, investigations into the appropriate timing, makeup (Pure PRP, Leukocyte and PRP, Pure PRF, Leukocyte and PRF), and concentration of PRP need to be further investigated. In place of the increasingly promising data in adult patients, further investigations need to be conducted in pediatric patients to determine whether PRP will have any considerable benefit.

\section{Conflicts of Interest}

We declare that we have no conflicts of interest in the authorship or publication of this article.

\section{Author Contribution Statement}

Authors Jorge Benito, Phong Truong, Eric Bradley, Cindy Ho, and Cameron McLaury contributed to the article's conception or design; drafted or critically revised the article; approved the final version to be published and agreed to be accountable for all aspects of the article.

\section{References}

1. Hall MP, Band PA, Meislin RT, Jazrawi LM, Cardone DA, et al. (2009) Platelet-rich plasma: Current concepts and application in sports medicine. J Am Acad Orthop Surg 17(10): 602-608.

2. Bray CC, Walker CM, Spence DD (2017) Orthobiologics in Pediatric Sports Medicine. Orthop Clin North Am 48(3): 333-342.

3. kalyani p, Kaarthikeyan g, Santhosh Kumar MP (2020) View of Platelet Rich Plasma-Platelet Counts and Application-A Literature Review. Journal of Pharmaceutical Research International 32(16): 131-138.

4. Cavallo C, Filardo G, Mariani E, Elizaveta K, Maurilio M, et al. (2014) Comparison of platelet-rich plasma formulations for cartilage healing: An in vitro study. J Bone Jt Surg - Ser A 96(5): 423-429.

5. Zhou Y, Zhang J, Wu H, Hogan MC V, Wang JHC, et al. (2015) The differential effects of leukocyte-containing and pure platelet-rich plasma (PRP) on tendon stem/progenitor cells - implications of PRP application for the clinical treatment of tendon injuries. Stem Cell Res Ther 6(1): pp. 173.

6. Le ADK, Enweze L, DeBaun MR, Dragoo JL (2018) Current Clinical Recommendations for Use of Platelet-Rich Plasma. Curr Rev Musculoskelet Med 11(4): 624-634.
7. Chahla J, Cinque ME, Piuzzi NS, Sandeep M, Andrew GG, et al. (2017) A Call for Standardization in Platelet-Rich Plasma Preparation Protocols and Composition Reporting: A Systematic Review of the Clinical Orthopaedic Literature. J Bone Jt Surg - Am 99(20): 1769-1779.

8. Miller LE, Parrish WR, Roides B, Bhattacharyya S (2017) Efficacy of platelet-rich plasma injections for symptomatic tendinopathy: Systematic review and meta-analysis of randomised injection-controlled trials. BMJ Open Sport Exerc Med 3(1): e000237.

9. LaPrade RF, Goodrich LR, Phillips J, Grant J Dornan, Travis Lee Turnbull et al. (2018) Use of Platelet-Rich Plasma Immediately After an Injury Did Not Improve Ligament Healing, and Increasing Platelet Concentrations Was Detrimental in an In Vivo Animal Model. Am J Sports Med 46(3): 702-712.

10. Schroeder AN, Dawn Comstock R, Collins CL, Everhart J, Flanigan D, et al. (2015) Epidemiology of overuse injuries among high-school athletes in the united states. J Pediatr 166(3): 600-606.

11. Fabricant PD, Kocher MS (2017) Management of ACL Injuries in Children and Adolescents. J Bone Joint Surg Am 99(7): 600-612.

12. Best TM, Caplan A, Coleman M, Laurie G, Jason H, et al. (2017) Not missing the future: A call to action for investigating the role of regenerative medicine therapies in pediatric/adolescent sports injuries. Curr Sports Med Rep 16(3): 202-210.

13. Hamid MS, Mohamed Ali MR, Yusof A, George J, Lee LPC, et al. (2014) Platelet-rich plasma injections for the treatment of hamstring injuries: A randomized controlled trial. Am J Sports Med. 2014;42(10): 2410-2418.

14. Figueroa D, Figueroa F, Calvo R, Vaisman A, Ahumada X, et al. (2015) Platelet-rich plasma use in anterior cruciate ligament surgery: Systematic review of the literature. Arthrosc - J Arthrosc Relat Surg 31(5): 981-988.

15. Podesta L, Crow SA, Volkmer D, Bert T, Yocum LA, et al. (2013) Treatment of partial ulnar collateral ligament tears in the elbow with platelet-rich plasma. Am J Sports Med 41(7): 1689-1694.

16. Chauhan A, McQueen P, Chalmers PN, Michael GC, Christopher LC, et al. (2019) Nonoperative Treatment of Elbow Ulnar Collateral Ligament Injuries With and Without Platelet-Rich Plasma in Professional Baseball Players: A Comparative and Matched Cohort Analysis. Am J Sports Med. 47(13): 3107-3119.

17. Sánchez M, Anitua E, Azofra J, Andía I, Padilla S, et al. (2007) Comparison of surgically repaired Achilles tendon tears using platelet-rich fibrin matrices. Am J Sports Med 35(2): 245-251.

18. Kon E, Filardo G, Delcogliano M, Mirco LP, Alessandro R, et al. (2009) Platelet-rich plasma: New clinical application. A pilot study for treatment of jumper's knee. Injury 40(6): 598-603.

19. Samy AM (2016) The role of platelet rich plasma in management of fracture neck femur: new insights. Int Orthop 40(5): 1019-1024.

20. Singh R, Rohilla R, Gawande J, Kumar Sehgal P (2017) To evaluate the role of platelet-rich plasma in healing of acute diaphyseal fractures of the femur. Chinese J Traumatol-English 20(1): 39-44. 
CC (P) This work is licensed under Creative

To Submit Your Article Click Here: $\quad$ Submit Article

DOI: $10.32474 /$ OSMOAJ.2021.05.000201

$\begin{gathered}\text { Orthopedics and Sports Medicine } \\ \text { Open Access Journal }\end{gathered}$
Assets of Publishing with us
- Global archiving of articles
- Immediate, unrestricted online access
- Rigorous Peer Review Process
- Authors Retain Copyrights
Orthopedics And Sports Medicine:
Open Access Journal

